\title{
Estrés académico en los estudiantes de la Licenciatura en Música de la Universidad de Sonora
}

M.M. María del Rocío Terán Díaz Landa

Universidad de Sonora

rocio.terandl@gmail.com

\section{Introducción}

En el 2011, varios docentes de la Licenciatura en Música de la Universidad de Sonora, observaron una elevada preocupación por parte de los estudiantes en relación a sus labores académicas. Los propios alumnos manifestaban continuamente su disgusto frente a ciertas situaciones y actividades universitarias que los sumían en un estado de ansiedad e inquietud constante, lo cual repercutía no solo en su rendimiento académico sino también en su salud y bienestar general. Este hecho fomentó, en ese mismo año, el interés por realizar un primer acercamiento al estudio del estrés en los estudiantes de música, evaluando algunos factores generadores de estrés, el grado en que se presentan y las diferencias que se aprecian según el género. Cuatro años después se realiza el mismo estudio, de manera más amplia, con el propósito de observar el mantenimiento o los cambios de dichos factores, con la finalidad de ofrecer tanto a docentes como a estudiantes un panorama general, a través de un estudio cuantitativo, que conlleve a la búsqueda de estrategias encaminadas a disminuir los agentes generadores de estrés.

\section{Desarrollo}

De acuerdo con la neurología, el estrés es una respuesta ante la homeostasis amenazada por un evento aversivo ambiental o biológico (Valencia-Alfonso, Feria-Velasco, Luquín, Díaz-Burke y García-Estrada, 2004). Es decir, el estrés perturba el equilibrio del ser humano provocando cambios significativos en el sistema nervioso, mismos que pueden manifestarse en el comportamiento y en la salud (Valencia y otros, 2004). Sarafino (1990, citado en Tolentino, 2009, p.8) ofrece una definición que deja en claro la condición que genera el estrés, argumentando que "resulta cuando las transacciones entre la persona y el medio conducen al individuo a la percepción de una discrepancia - real o ficticia - entre las demandas de la situación y los recursos psicológicos, biológicos o sociales de los que no dispone".

En la actualidad, el estrés ha llegado a afectar a gran parte de la población, motivo por el cual han surgido diversas investigaciones dedicadas a buscar las fuentes de su origen, consecuencias, estilos y estrategias para afrontarlo. Así mismo, se han encontrado diferentes tipos de estrés - que dependen de diversos factores - cuyos estudios no han sido del todo equitativos (Polo, Hernández y Poza, 2001).

A decir de Monzón (2007), el denominado estrés académico o del estudiante no ha recibido la atención suficiente. Dicho estrés se define como aquél que se produce en el ámbito educativo y que puede llegar a afectar tanto a profesores como a estudiantes. Estudios recientes afirman que el nivel de estrés en los alumnos universitarios ha ido en aumento, hecho que repercute, en gran medida, en el rendimiento académico y en la salud del individuo (Burgos y Marín, 2010). Entre las principales causas que originan el estrés 
en los estudiantes se encuentran los períodos de exámenes, la sobrecarga académica, una mala organización y la falta de tiempo. Así mismo, se observa que frecuentemente los alumnos universitarios desarrollan hábitos nocivos para la salud. Este hecho está determinado por una crisis económica y social, que se traduce en factores de presión, y por ende de estrés. Es preciso señalar el mundo competitivo en el cual el estudiante está inmerso, aunado a los problemas personales y de la vida cotidiana. Autores como Cassaretto, Chau, Oblitas y Valdez (2003) señalan que las fuentes y niveles de estrés responden a las condiciones socioeconómicas del país y a la etapa de desarrollo en la que los jóvenes se encuentran, donde el logro personal y social depende mucho de la realización profesional y donde la incertidumbre de vivir en un país con condiciones económicas variables, los enfrenta a sentimientos causantes de angustia y malestar (Cassaretto, Chau, Oblitas y Valdez, 2003). Por lo tanto, el nivel de estrés aumenta cuando existe una evidente preocupación por el futuro.

Indudablemente, todos los aspectos antes mencionados influyen de un modo $u$ otro en los estudiantes de música, sin embargo, el estudio de dicho arte conlleva a otros aspectos que dan origen al estrés (Calatayud, 2010).

Resulta curioso observar como la música puede provocar dos efectos totalmente contradictorios. Por un lado, como oyente y como forma de terapia ocupacional, es considerada un medio eficaz para reducir el estrés y lograr la relajación. Por otro lado su estudio a nivel profesional puede conducir a los estudiantes a padecer un alto nivel de estrés y ansiedad. Helen Jane Orzel (2010) refiere que en la atmósfera universitaria de música el estrés y el desgaste prevalecen y son aceptados como parte de la cultura. Los estudiantes frecuentemente sufren alteraciones de conducta debido al estrés y la ansiedad a la que están sometidos antes de una interpretación e incluso pueden llegar a sufrir depresión si algo no resulta de manera satisfactoria. Guillermo Dalia Cirujeda (2004) señala que es un hecho comprobado que los denominados nervios o la ansiedad frente a una ejecución musical interfieren de manera negativa.

Los estudios de prevalencia (Tineo, 2008) indican que entre el 40 y $70 \%$ de los estudiantes de música afirman que la ansiedad escénica les afecta negativamente disminuyendo su rendimiento y la calidad global de su ejecución. De ellos, sobre un porcentaje que se sitúa en torno al 20\% la ansiedad les afecta de manera significativa. Aparecen pensamientos inadecuados antes, durante y después de la actuación que fomentan y a veces mantienen los problemas de ansiedad.

Sin embargo, la ansiedad y el estrés son elementos que siempre están presentes en la vida de los músicos, ya que su interpretación es el resultado de un largo período de trabajo. Incluso se considera benéfico presentar cierto nivel de miedo escénico para alcanzar el éxito, ya que, como señalan Hamann y Sobaje (1983, citado en Tineo, 2008), la ansiedad puede mejorar el logro intelectual y físico si se maneja de manera adecuada. Este hecho se explica científicamente con la ley de Yerkes Dodson que afirma que existe una estrecha relación entre el rendimiento y la motivación, es decir, un buen rendimiento se consigue con cierto nivel de motivación, el cual aumenta si se presenta cierto grado de estrés (Tineo, 2008).

Recientes investigaciones en San Jose State University (Orzel, 2010) han evaluado el nivel de estrés en los estudiantes universitarios de música cuyos resultados fueron los siguientes: el nivel de estrés aumenta por la sobrecarga de trabajos, el 
tiempo límite de entrega de trabajos, el tipo de trabajos que piden los profesores y las evaluaciones. Así mismo se observó que varios estudiantes presentan desgaste y ansiedad por los compromisos musicales que adquieren y por la falta de tiempo para dedicarse a otras actividades. Por último se hizo notar que el nivel de estrés varía según el área de especialización y el sexo.

Otro trabajo realizado en la Universidad de Madrid (Tineo, 2008) expone que las situaciones de mayor estrés que presentan los estudiantes en general tienen relación con la falta de tiempo para poder cumplir las actividades académicas y con la sobrecarga académica, lo cual manifiesta que no son las situaciones de evaluación las que generan más estrés sino la relación que existe entre la cantidad de trabajo y el tiempo.

Como se mencionó anteriormente, el presente trabajo evalúa algunos factores generadores de estrés en los alumnos de la Licenciatura en música de la Universidad de Sonora, el grado en el que se presentan y la diferencia que se observa en cuanto al género. Así mismo, se realiza una comparación con los resultados obtenidos en el estudio realizado en el año 2011.

\section{Método}

Participantes

En el 2011 se recopilaron datos de una muestra aleatoria de 20 estudiantes de la Licenciatura en Música de la Universidad de Sonora, lo cual correspondía al 24.3\% de la población total (82 alumnos), siendo el $50 \%$ varones y el $50 \%$ mujeres.

A principios del 2015 se aplicó el estudio, en la misma licenciatura, al $50.4 \%$ de la población estudiantil (51 alumnos) que se encontraba cursando de manera regular los semestres II, IV, VI y VIII, siendo el $45 \%$ de sexo femenino y el $55 \%$ masculino.

\section{Procedimiento}

La aplicación del cuestionario se realizó de manera colectiva (a excepción de 4 estudiantes que lo respondieron en otro momento) y dentro del aula de clases. Se dieron instrucciones escritas, incluidas en el cuestionario, y verbales para clarificar el procedimiento.

No se estableció un tiempo límite para responder el cuestionario. El tiempo empleado por los estudiantes fluctuó entre 8 y 15 minutos.

\section{Instrumento}

El instrumento de medida utilizado fue el Inventario de Estrés Académico (I. E. A.; Polo, Hernández y Poza, 1996) diseñado específicamente para evaluación del estrés en universitarios. Dicho inventario está formado por 11 ítems e incluye situaciones potencialmente generadoras de estrés en los estudiantes dentro del ámbito académico. Tales situaciones son las siguientes:

1. Realización de un examen

2. Exposición de trabajos en clase

3. Intervención en el aula

4. Subir al despacho del profesor en horas de tutorías

5. Sobrecarga académica.

6. Masificación de las aulas

7. Falta de tiempo para poder cumplir con las actividades académicas

8. Competitividad entre compañeros

9. Realización de trabajos obligatorios para aprobar la asignatura

10. La tarea de estudio

11. Trabajar en grupo

Para el estudio del 2015 se agregó al I. E. A. un factor más generador de estrés, debido a las características propias de la Licenciatura en Música y sus necesidades: 
12. presentaciones musicales en público Para cada una de las situaciones el estudiante pudo responder de acuerdo a una escala tipo Likert cuyos valores se encuentran de 1 a 5, en donde 1 representa Nada de estrés, 2 Poco estrés, 3 Estrés moderado, 4 Estrés y 5 Mucho estrés, lo cual indica el grado de estrés que dicha situación puede provocar.

\section{Resultados}

Los resultados obtenidos en el 2011 muestran las respuestas de los estudiantes que corresponden a cada uno de los 11 ítems contenidos en el instrumento de evaluación. De acuerdo con ellos se obtuvo la medida de tendencia central correspondiente a la mediana (Tabla 1) y la diferencia en cuanto al sexo (Tabla 2).

Tabla 1. Escala Factores generadores de estrés 2011

\begin{tabular}{lllll}
\hline Item & Núm & Mín & Máx & Mediana \\
\hline & & & & \\
1. Realización de un examen & 20 & 1 & 5 & 3 \\
2. exposición de trabajos en clase & 20 & 1 & 5 & 2.5 \\
3. Intervención en el aula & 20 & 1 & 5 & 1.5 \\
4. Subir al despacho del profesor & 20 & 1 & 5 & 1 \\
5. Sobrecarga académica & 20 & 1 & 5 & 4 \\
6. Masificación del aula & 20 & 1 & 5 & 2 \\
7. Falta de tiempo para poder cumplir con las & 20 & 1 & 5 & 4.5 \\
actividades académicas & & & & \\
8. Competitividad entre compañeros & 20 & 1 & 5 & 1.5 \\
9. Realización de trabajos obligatorios para aprobar & 20 & 1 & 5 & 3.5 \\
asignatura & & & & \\
10. La tarea de estudio & 20 & 1 & 5 & 2 \\
11. Trabajar en grupo & 20 & 1 & 5 & 3
\end{tabular}

Tabla 2. Escala Factores generadores de estrés en hombres y mujeres 2011

\begin{tabular}{|c|c|c|c|c|c|}
\hline Item & Núm & Mín & Máx & $\begin{array}{l}\text { Mediana } \\
\text { Mujeres }\end{array}$ & $\begin{array}{l}\text { Mediana } \\
\text { Hombres }\end{array}$ \\
\hline 1. Realización de un examen & 20 & 1 & 5 & 4 & 3 \\
\hline 2. exposición de trabajos en clase & 20 & 1 & 5 & 2 & 2.5 \\
\hline 3. Intervención en el aula & 20 & 1 & 5 & 1.5 & 1.5 \\
\hline 4. Subir al despacho del profesor & 20 & 1 & 5 & 1 & 1 \\
\hline 5. Sobrecarga académica & 20 & 1 & 5 & 4.5 & 4 \\
\hline 6. Masificación del aula & 20 & 1 & 5 & 3 & 1 \\
\hline $\begin{array}{l}\text { 7. Falta de tiempo para poder cumplir con } \\
\text { las actividades académicas }\end{array}$ & 20 & 1 & 5 & 5 & 4 \\
\hline 8. Competitividad entre compañeros & 20 & 1 & 5 & 2 & 1 \\
\hline $\begin{array}{l}\text { 9. Realización de trabajos obligatorios } \\
\text { para aprobar asignatura }\end{array}$ & 20 & 1 & 5 & 3.5 & 3 \\
\hline 10. La tarea de estudio & 20 & 1 & 5 & 2.5 & 2 \\
\hline 11. Trabajar en grupo & 20 & 1 & 5 & 3 & 2 \\
\hline
\end{tabular}


Como puede apreciarse en las tablas anteriores, los resultados de los alumnos informan que las situaciones que mayor estrés les produce son la falta de tiempo para cumplir con las actividades académicas y la sobrecarga académica; en segundo término se observa la realización de un examen y la de trabajos obligatorios para aprobar una asignatura. Como situaciones de menor estrés se encuentran el trabajo en grupo, la exposición de trabajos en clase, la tarea de estudio y la competitividad entre compañeros; y, finalmente se presentan valores inferiores que corresponden a la intervención en el aula (responder a una pregunta del profesor, realizar preguntas), a la masificación del aula y a subir al despacho del profesor en horas de tutorías.

Las siguiente gráfica muestra los resultados obtenidos, en los cuales se observa que en general el sexo femenino presenta un estrés mayor en comparación con el sexo masculino (Figura 1).

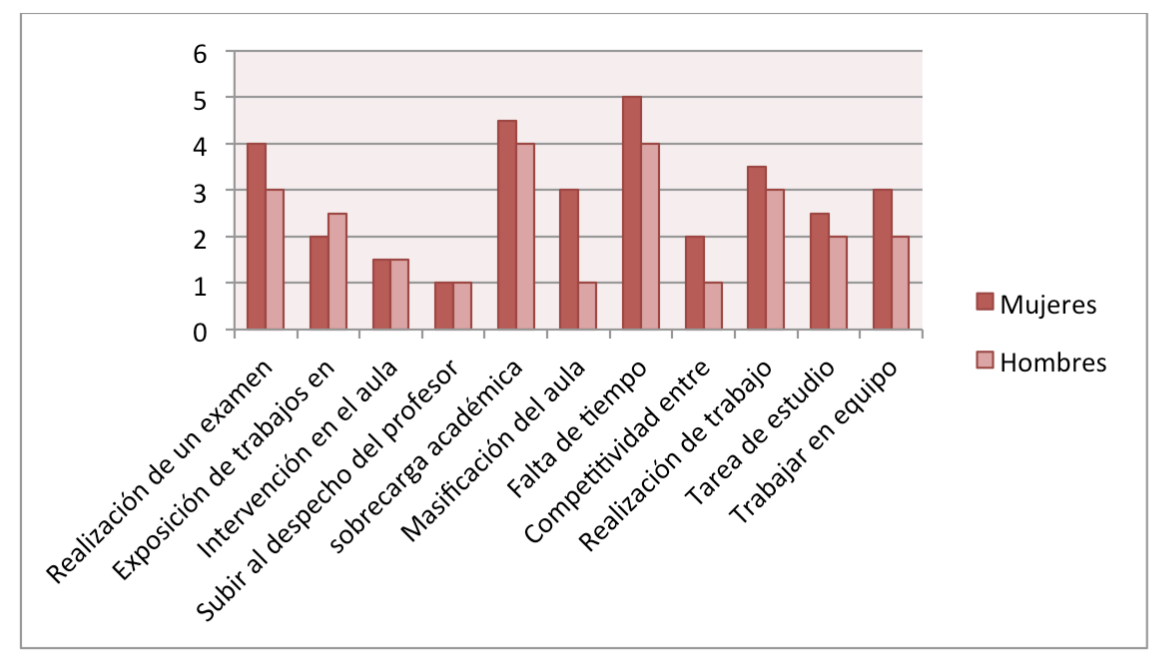

Figura 1. Factores de estrés en estudiantes de la Licenciatura en Música de la Universidad de Sonora, hombres y mujeres (2011).

A continuación se observa (Tabla 3 y Figura 2) el nivel de los factores generadores de estrés en el 2015 y las diferencias que se aprecian tanto en hombres como en mujeres. 
Tabla 3. Escala Factores generadores de estrés en hombres y mujeres 2015

Núm Mín Máx Mediana Mediana

Item

Mujeres Hombres

1. Realización de un examen

2. exposición de trabajos en clase

3. Intervención en el aula

4. Subir al despacho del profesor

5. Sobrecarga académica

6. Masificación del aula

7. Falta de tiempo para poder cumplir con las actividades académicas

8. Competitividad entre compañeros

9. Realización de trabajos obligatorios para aprobar asignatura

10. La tarea de estudio

11. Trabajar en grupo

12. Presentaciones musicales en público
51

51

51

51

51

51

51

51

1

1

51

1

$51 \quad 1$

51

5

3

3

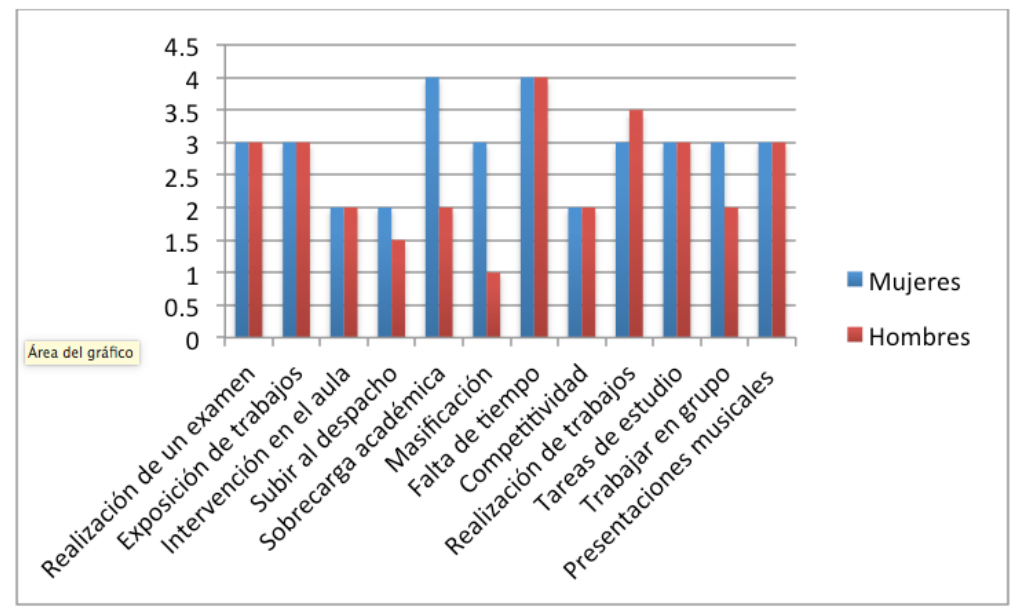

Figura 2. Factores de estrés en los estudiantes de la Licenciatura en Música de la Universidad de Sonora, hombres y mujeres (2015). 
Al igual que en el 2011, los resultados obtenidos en el 2015 muestran que los dos factores de estrés con mayor nivel en los estudiantes de música son la falta de tiempo para cumplir con las actividades académicas y la sobrecarga académica. De igual manera y en el mismo nivel, le siguen la realización de un examen, la realización y la exposición de trabajos obligatorios para aprobar la asignatura, la tarea de estudio, el trabajo en equipo y las presentaciones musicales en público. En menor grado aparece la intervención en el aula, subir al despacho del profesor, la masificación del aula y la competitividad entre compañeros (Figura 3).

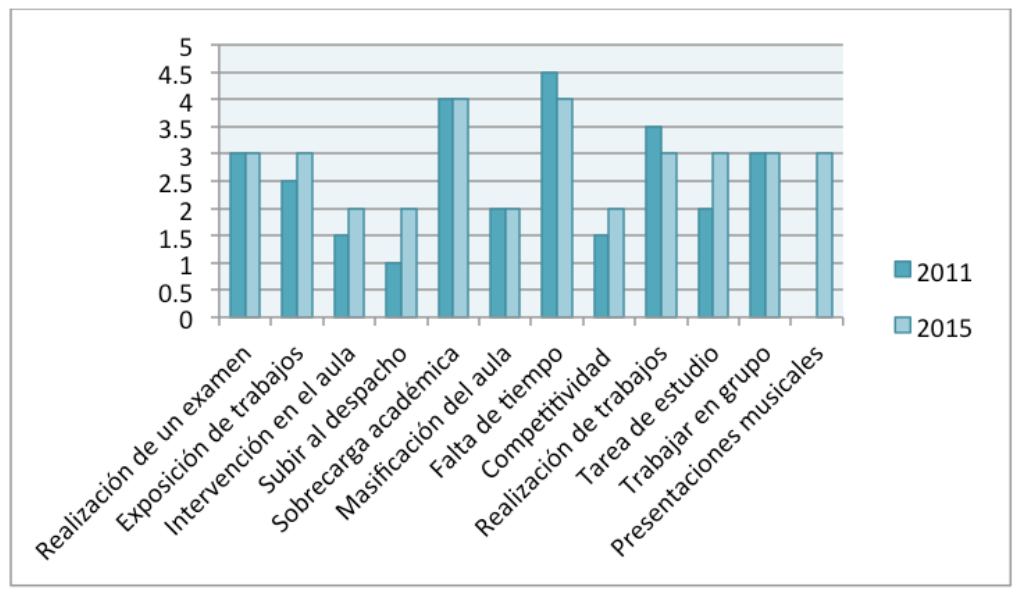

Figura 3. Factores de estrés en los estudiantes de la Licenciatura en Música de la Universidad de Sonora, 2011 y 2015.

Por último, al estudiar las diferencias en el nivel de estrés que se presentan en relación al semestre que se está cursando (Figura 4), se encontró que en todos pre- valece en primer lugar la sobrecarga académica y la falta de tiempo, con un grado mayor durante el VI semestre.

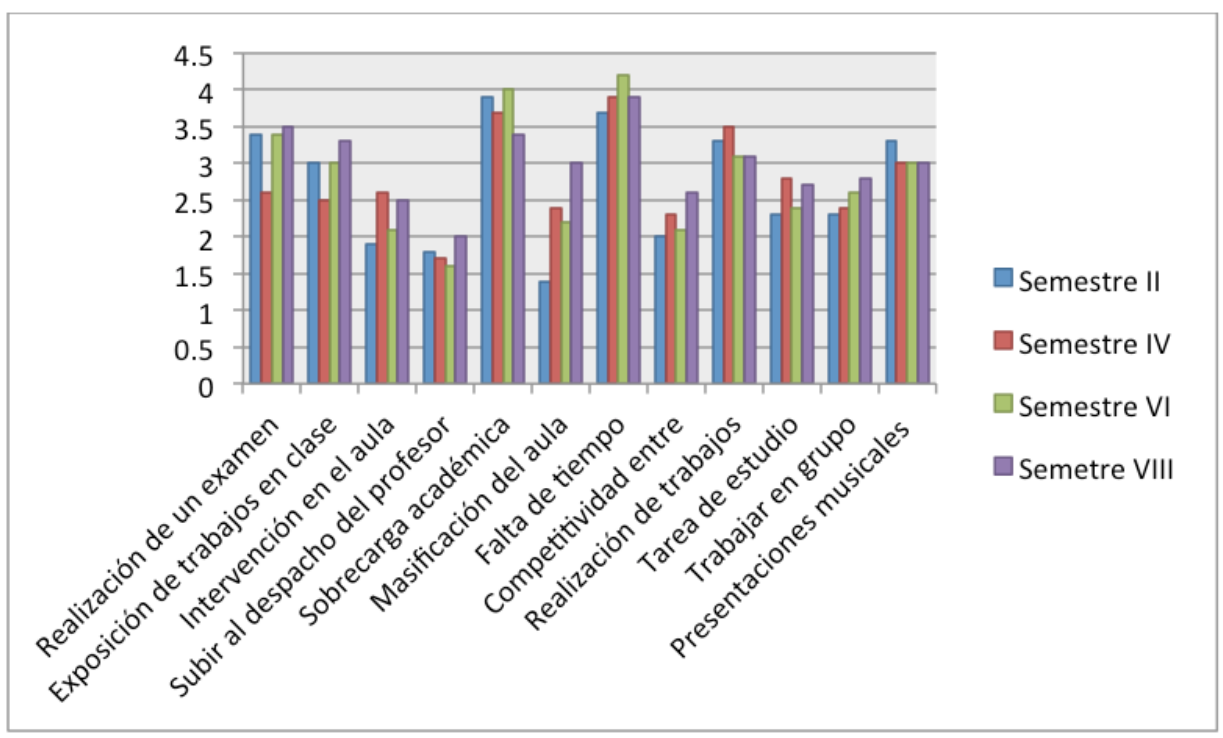

Figura 4. Factores de estrés en los estudiantes de la Licenciatura en Música de la Universidad de Sonora, semestres II, IV, VI y VIII de 2015. 


\section{Conclusiones}

Contrariamente a lo que se cree, las situaciones de evaluación y las presentaciones en público no son las que generan más estrés en los estudiantes de música sino la relación que se da entre el tiempo y el trabajo. Es decir, los alumnos sienten mayor presión al momento de tener sobrecarga académica y falta de tiempo para poder cumplir con las actividades académicas, a pesar de presentar no solo evaluaciones teóricas sino también prácticas -relacionadas con la ejecución musical -.

$\mathrm{Al}$ hacer una distinción de género, resulta interesante observar que las mujeres presentan un mayor índice de estrés que los hombres en casi todas las situaciones presentadas en el I.E.A., a excepción de la realización y exposición de trabajos en clase y la tarea de estudio. Este hecho ofrece un marco de referencia para ela- borar diversas herramientas y estrategias que ayuden a disminuir el estrés de acuerdo al género.

Queda para futuras investigaciones conocer el motivo por el cuál las mujeres manifiestan un mayor índice de estrés en el ámbito académico que los hombres, llevar a cabo estudios que expliquen las razones del porqué de los cambios en el nivel de estrés en los diferentes semestres de la licenciatura así como también una revisión curricular de la misma e ir más allá de las investigaciones cuantitativas con la finalidad de ofrecer estrategias factibles y eficaces que reduzcan los índices de estrés y logren mejoras en la calidad del aprendizaje, la salud y el bienestar de los futuros artistas.

Bibliografía

Burgos Monago, E. y Marín Ferrara, J. M. (2010). Estrés académico en los estudiantes universitarios, Facultad de Ciencias Económicas Universidad de Extremadura. Recuperado el 6 de mayo de 2011 de http://es.scrib.com/doc/28105721/Estres-academico-en-los-estudiantes-universitarios

Calatayud Torres, A. (2010). Ansiedad escénica en músicos: un modelo teórico-práctico. Recuperado el 5 de mayo de 2011 de http://www.cetecova.com/index.php/ revista-correo-conductual-coco/9//33-ansiedad-escenica-en-musicosun-modelo-teorico-practico

Cassareto, M., Chau, C., Oblitas, H. Y Valdez, N. (2003). Estrés y afrontamiento en estudiantes de psicología. Revista de Psicología de la PUCP, vol. XXI. Recuperado el 5 de mayo de 2011 de https://dialnet.unirioja.es/descarga/articulo/994031.pdf

Dalia Cirujeda, G. (2004). Ansiedad Escénica. Madrid: Mundimúsica Ediciones, S. L.

Monzón, M. (2007). Estrés académico en estudiantes universitarios. Apuntes de Psicología. Recuperado el 5 de mayo de 2011 de http://www.cop.es/delegaci/andocci/files/contenidos/VOL25_1_7.pdf

Orzel, H. J. (2010). Undergraduate Music Students stress and burnout. Master's Theses, Peper 3887. Recuperado el 6 de mayo de 2011 de http://scholarworks.sjsu.edu/etd_theses/3887

Polo, A., Hernández, J. M. y Poza, C. (2001). Evaluación del estrés académico en estudiantes universitarios. Universidad Autónoma de Madrid. Recuperado el 5 de mayo de 2011 de http:/www.unilean.es/estudiantes/atencion_universitario/ articulo.pdf 
Tineo Guerrero, V. (2008). Trastornos asociados al entrenamiento musical. Recuperado el 4 de mayo de 2011 de http://www.relafare.eu.html_articulos/31-trastornosasociados.html

Tolentino, S. (2009). Perfil de estrés académico en alumnos de licenciatura en psicología, de la Universidad Autónoma de Hidalgo en la Escuela Superior de Actopan. Tesis. Recuperado el 21 de enero de 2015 de http://www.uaeh.edu.mx/nuestro_alumnado/esc_sup/actopan/licenciatura/Perfil\%20de\%20estres\%20academico\%20en\%20alumnos.pdf

Valencia-Alfonso, C. E., Feria-Velasco, A., Luquín, S., Díaz-Burke, Y. y García-Estrada, J. (2004). Efectos cerebrales del medio ambiente. Recuperado el 3 de mayo de 2011 de http://www.psiquiatria.com/articulos/ansiedad/estres/etiologia445/17459/

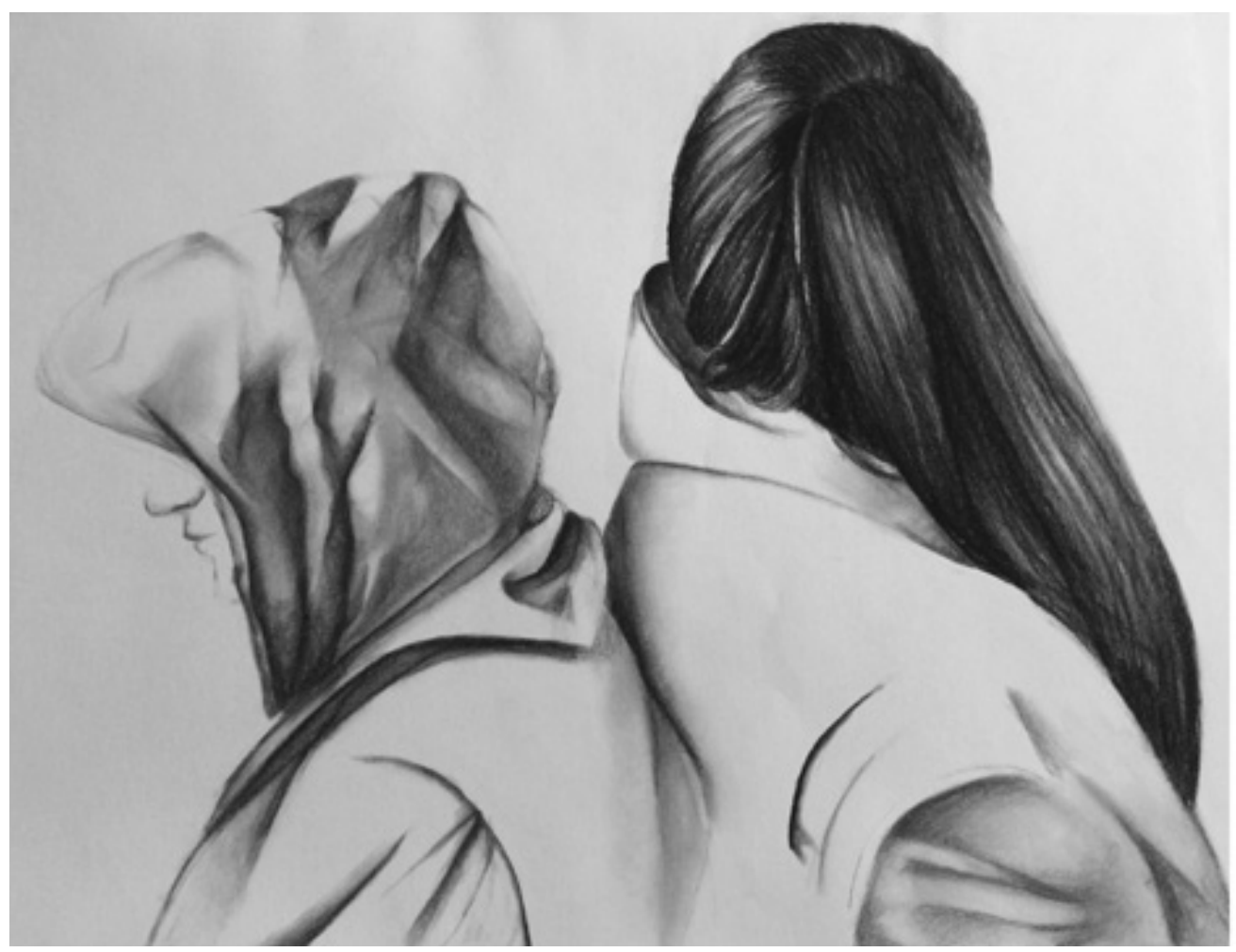

